PROCEEDINGS OF THE

AMERICAN MATHEMATICAL SOCIETY

Volume 127, Number 10, Pages 3081-3090

S 0002-9939(99)04867-4

Article electronically published on April 23, 1999

\title{
AN EXTENSION OF THE WORK OF V. GUILLEMIN ON COMPLEX POWERS AND ZETA FUNCTIONS OF ELLIPTIC PSEUDODIFFERENTIAL OPERATORS
}

\author{
BOGDAN BUCICOVSCHI
}

(Communicated by Peter Li)

\begin{abstract}
The purpose of this note is to extend the methods and results of Guillemin on elliptic self-adjoint pseudodifferential operators of order one, from operators defined on smooth functions on a closed manifold to operators defined on smooth sections in a vector bundle. The case of bundles of Hilbert modules of finite type over a finite von Neumann algebra will also be treated.
\end{abstract}

\section{INTRODUCTION}

Let $M$ be a closed Riemannian manifold of dimension $m$ and $E$ a vector bundle over $M$ endowed with a hermitian metric. The fibers of $E$ are finite dimensional vector spaces over $\mathbb{C}$ or, more general, finite type Hilbert modules over a von Neumann algebra $\mathcal{A}$. The first situation corresponds to the case $\mathcal{A}=\mathbb{C}$. Throughout this paper we will denote by $\Psi(E)$ or simply by $\Psi$ the algebra of classical pseudodifferential operators acting on smooth sections in $E$ (for the case when $\mathcal{A}$ is an arbitrary von Neumann algebra, see $[\mathrm{BFKM}]$ for definitions and properties). We will also denote by $\Psi^{s}(E)$ the subspace of pseudodifferential operators of complex order $s$. The total symbol $\sigma_{\text {total }}(x, \xi)$ of such an operator $A \in \Psi^{s}$ has locally an asymptotic expansion of the form:

$$
\sigma_{\text {total }}(x, \xi) \sim \sum_{k=0}^{\infty} \sigma_{s-k}(x, \xi)
$$

where $\sigma_{s-k}(x, \xi)$ are sections of the endomorphism bundle of the pull-back of $E$ with respect to the projection map $T^{*}(M) \backslash\{0\} \rightarrow M$. Each section $\sigma_{s-k}(x, \xi)$ is a homogeneous function in the variable $\xi$ of degree of homogeneity $s-k \in \mathbb{C}$, $\sigma_{s-k}(x, \lambda \xi)=\lambda^{s-k} \sigma_{s-k}(x, \xi)$ for any $\lambda>0$.

The space $C^{\infty}(E)$ of smooth sections of $E$ over $M$ has a canonical metric

$$
\langle f, g\rangle=\int_{M}\langle f(x), g(x)\rangle_{x} d v o l
$$

where $\langle\cdot, \cdot\rangle_{x}$ is the hermitian metric in the fiber above $x \in M$. The $L^{2}$ completion of $C^{\infty}(E)$ with respect to $\langle\cdot, \cdot\rangle$ will be denoted by $L^{2}(E)$. A pseudodifferential operator becomes an unbounded operator on $L^{2}(E)$.

Received by the editors December 1, 1997.

1991 Mathematics Subject Classification. Primary 58G25, 35P05.

(C)1999 American Mathematical Society 
We will consider now an elliptic pseudodifferential operator of order one $A \in \Psi^{1}$ which is self-adjoint and positive with respect to $\langle\cdot, \cdot\rangle$. Suppose that the spectrum of $A$ is included in the interval $(\epsilon, \infty)$ for a sufficiently small $\epsilon>0$. Then one can define the complex powers $A^{s}, s \in \mathbb{C}$ in the following way

$$
A^{s}=\frac{1}{2 \pi i} \int_{\gamma} \lambda^{s}(\lambda-A)^{-1} d \lambda \quad \text { when } \operatorname{Re}(s)<0
$$

(where $\gamma$ is a contour in the complex plane obtained by joining two lines parallel to the negative real axis by a circle around the origin) and

$$
A^{s}=A^{s-k} A^{k} \quad \text { for } \operatorname{Re}(s) \geq 0
$$

for large enough $k \in \mathbb{Z}$ so that $s-k$ is negative.

One of the goals of our paper is to show that $A^{s}$ is a pseudodifferential operator of complex order $s$. We remind the reader that this fact has been proven first by Seeley $[\mathrm{S}]$ in the case of finite dimensional hermitian bundle $E$ and extended to the case of von Neumann bundles in [BFKM]. Guillemin has provided an alternative approach in $[\mathrm{G}]$; his treatment covers essentially the case of pseudodifferential operators acting on smooth functions on $M$. We will show how one can adjust his strategy to the case of operators acting on sections in a vector bundle $E$. The main difficulty arises from the fact that the algebra of endomorphisms of $E$ is noncommutative (fiberwise it is equal to the algebra of the $\mathcal{A}$-invariant endomorphisms of the fiber, as compared to Guillemin's case where the fiber is isomorphic to $\mathbb{C}$ ). In the same spirit of Guillemin, we will show that the zeta function of $A$ defined as:

$$
\zeta_{A}(s)=\operatorname{Trace}_{N}\left(A^{s}\right) \quad \text { for } \operatorname{Re}(s)<-m
$$

has a meromorphic extension over the complex plane $\mathbb{C}$ with at most simple poles at $-m,-m+1, \ldots$. The residue of $\zeta_{A}$ at $-m$ will be equal to a quantity that depends only on the principal symbol $\sigma_{1}$ of the operator $A$.

Throughout the paper $A$ will be a classical pseudodifferential operator of order 1 . The case of an operator of any other positive order can be reduced to the case in which the order is equal to 1.

I thank Prof. D. Burghelea for his support and suggestions and Prof. T. Kappeler and L. Friedlander for useful discussions.

\section{COMPLEX POWERS OF PSEUDODIFFERENTIAL OPERATORS}

The goal of the section is proving the following:

Theorem 1.1. Let $A$ be an elliptic, positive, self-adjoint pseudodifferential operator of order one. Suppose that $\operatorname{Spec}(A) \in(\epsilon, \infty)$ for a sufficiently small $\epsilon>0$. Then its complex powers $A^{s}$, defined as in (0.1) and (0.2), are pseudodifferential operators of order $s \in \mathbb{C}$.

To show this we will need the following:

Proposition 1.2. There exists a holomorphic family of pseudodifferential operators $A_{s}$ for $s \in \mathbb{C}$ such that $A_{0}=I d, A_{s} A_{t}=A_{s+t}$ and the difference $A_{1}-A$ is a smoothing operator.

$\left(A_{s}\right)_{s \in \mathbb{C}}$ can be thought of as an approximation of the powers of $A$ that lie inside $\Psi$. We will show that $A_{s}-A^{s}$ are smoothing operators. Then Theorem 1.1 becomes a straightforward corollary of Proposition 1.2. 
To construct the family $\left(A_{s}\right)_{s \in \mathbb{C}}$ it will be convenient to consider the cohomology of the group $(\mathbb{C},+)$ with coefficients in the representation of $(\mathbb{C},+)$ on the space of sections $C^{\infty}(\operatorname{End}(\tilde{E}))$. Here $\tilde{E}$ is the pull-back of the initial vector bundle $E$ over $M$ with respect to the projection map of the cosphere bundle $S^{*}(M) \rightarrow M$. This construction generalizes the cohomology considered by Guillemin in [G] for the trivial representation of $(\mathbb{C},+)$ on the space of smooth functions on $S^{*}(M)$.

Let $\sigma$ be a fixed section $\sigma: S^{*}(M) \rightarrow \operatorname{End}(\tilde{E})$ so that $\sigma(x, \xi): E_{x} \rightarrow E_{x}$ is an invertible positive self-adjoint endomorphism for any $(x, \xi) \in S^{*}(M)(\sigma$ will be the restriction of the principal symbol of $A$ to $\left.S^{*}(M)\right)$. The representation of $(\mathbb{C},+)$ on $C^{\infty}(\operatorname{End}(\tilde{E}))$ we consider is the following one: any $s \in \mathbb{C}$ acts on a section $g: S^{*}(M) \rightarrow \operatorname{End}(\tilde{E})$ by $s \cdot g=\sigma^{-s} g \sigma^{s}$.

Let $\mathcal{C}^{r}=\mathcal{C}^{r}\left(\mathbb{C} ; C^{\infty}(\operatorname{End}(\tilde{E}))\right.$ be the space of functions

$$
f: \underbrace{\mathbb{C} \times \mathbb{C} \times \cdots \times \mathbb{C}}_{r \text { times }} \rightarrow C^{\infty}(\operatorname{End}(\tilde{E}))
$$

that are smooth, $f(\cdot)(x, \xi): \mathbb{C} \times \mathbb{C} \times \ldots \mathbb{C} \rightarrow \operatorname{End}\left(E_{x}\right)$ are holomorphic for any fixed $(x, \xi) \in S^{*}(M)$ and $f\left(s_{1}, \ldots, s_{r}\right)=0$ if at least one $s_{i}$ is equal to zero.

Let $\delta^{r}: \mathcal{C}^{r} \rightarrow \mathcal{C}^{r+1}$ defined as:

$$
\begin{array}{r}
\left(\delta^{r} f\right)\left(s_{0}, s_{1}, \ldots, s_{r}\right)=s_{0} \cdot f\left(s_{1}, \ldots, s_{r}\right)+\sum_{i=1}^{r}(-1)^{i} \\
f\left(s_{0}, \ldots, s_{i-1}+s_{i}, \ldots, s_{r}\right) \\
+(-1)^{r+1} f\left(s_{0}, \ldots, s_{r-1}\right) .
\end{array}
$$

Let $\mathcal{H}^{r}\left(\mathbb{C} ; C^{\infty}(\operatorname{End}(\tilde{E}))\right)=\operatorname{Ker} \delta^{r} / \operatorname{Im} \delta^{r-1}$.

Proposition 1.3. $\mathcal{H}^{2}\left(\mathbb{C} ; C^{\infty}(\operatorname{End}(\tilde{E}))\right)=0$.

Moreover, for each 2-cocycle $f$ there exists a unique 1-cochain $h$ such that $\delta h=f$ and $h$ has a prescribed value at $1, h(1)$.

Proof. Let $f: \mathbb{C} \times \mathbb{C} \rightarrow C^{\infty}(\operatorname{End}(\tilde{E}))$ so that for all $a, b, c \in \mathbb{C}$

$$
\left\{\begin{array}{l}
f(0, b)=f(a, 0)=0, \\
\left(\delta^{2} f\right)(a, b, c)=a \cdot f(b, c)-f(a+b, c)+f(a, b+c)-f(a, b)=0 .
\end{array}\right.
$$

We will try to find $h: \mathbb{C} \rightarrow C^{\infty}(\operatorname{End}(\tilde{E}))$ such that

$$
\left(\delta^{1} h\right)(a, b)=\sigma^{-a} h(b) \sigma^{a}-h(a+b)+h(a)=f(a, b) .
$$

The existence of an $h$ as above implies:

$$
h^{\prime}(a)=\sigma^{-a} h^{\prime}(0) \sigma^{a}-\frac{\partial f}{\partial b}(a, 0) .
$$

Consider $h$ to be the unique solution of the previous equation with $h(0)=0$ and with a fixed prescribed value at $1, h(1) . h$ can be found in the following way:

Let $\Phi(t)$ be the automorphism of $C^{\infty}(\operatorname{End}(\tilde{E}))$ given by $A \rightarrow \sigma^{-t} A \sigma^{t}$. Then

$$
h(a)=-\int_{0}^{a} \frac{\partial f}{\partial b}(t, 0) d t+\int_{0}^{a} \Phi(t)\left(h^{\prime}(0)\right) d t .
$$


If $T(a) A=\int_{0}^{a} \Phi(t) A d t$, then, in order to get any prescribed value for $h(1)$, we need to show that $T(1)$ is surjective. Indeed, we have:

$$
\begin{aligned}
T(1) A & =\int_{0}^{\frac{1}{2}} \sigma^{-t} A \sigma^{t} d t+\int_{\frac{1}{2}}^{1} \sigma^{-t} A \sigma^{t} d t \\
& =T\left(\frac{1}{2}\right) A+\Phi\left(\frac{1}{2}\right) T\left(\frac{1}{2}\right) A=\left(\operatorname{Id}+\Phi\left(\frac{1}{2}\right)\right) T\left(\frac{1}{2}\right) A
\end{aligned}
$$

and by induction

$$
T(1) A=\left(\operatorname{Id}+\Phi\left(\frac{1}{2}\right)\right)\left(\operatorname{Id}+\Phi\left(\frac{1}{4}\right)\right) \ldots\left(\operatorname{Id}+\Phi\left(\frac{1}{2^{n}}\right)\right) T\left(\frac{1}{2^{n}}\right) A .
$$

But the map $A \rightarrow 2^{n} \int_{0}^{\frac{1}{2^{n}}} \sigma^{-t} A \sigma^{t} d t$ is close to the identity for a sufficiently large $n$ so $T\left(\frac{1}{2^{n}}\right)$ is invertible. $\left(\operatorname{Id}+\Phi\left(\frac{1}{2^{i}}\right)\right)$ is invertible as well, because $\Phi(t)$ is positive self-adjoint in any fiber above $(x, \xi) \in S^{*}(M)$, for any real $t$.

Thus we obtain a continuous map $h: \mathbb{C} \rightarrow C^{\infty}(\operatorname{End}(\tilde{E}))$ that is holomorphic in all fibers $E_{(x, \xi)}$ and $h \in \mathcal{C}^{1}$. We will show that $\delta h=f$ so $f$ is a coboundary. To see this, let

$$
g(a, b)=f(a, b)-\left(\sigma^{-a} h(b) \sigma^{a}-h(a+b)+h(a)\right) .
$$

Clearly $\delta h=f$ if and only if $g \equiv 0$. Denote by $\frac{\partial}{\partial b}$ the partial derivative with respect to the second variable. Then:

$$
\frac{\partial g}{\partial b}(a, b)=\frac{\partial f}{\partial b}(a, b)-\sigma^{-a} h^{\prime}(b) \sigma^{a}+h^{\prime}(a+b) .
$$

From (1.1) we get:

$$
\begin{aligned}
h^{\prime}(b) & =\sigma^{-b} h(0) \sigma^{b}-\frac{\partial f}{\partial b}(b, 0) \quad \text { and } \\
h^{\prime}(a+b) & =\sigma^{-(a+b)} h^{\prime}(0) \sigma^{(a+b)}-\frac{\partial f}{\partial b}(a+b, 0) .
\end{aligned}
$$

These two equalities and (1.2) imply

$$
\begin{aligned}
\frac{\partial g}{\partial b}(a, b)= & \frac{\partial f}{\partial b}(a, b)-\sigma^{-a}\left(\sigma^{-b} h^{\prime}(0) \sigma^{b}-\frac{\partial f}{\partial b}(b, 0)\right) \sigma^{a}+\sigma^{-(a+b)} h^{\prime}(0) \sigma^{(a+b)} \\
& -\frac{\partial f}{\partial b}(a+b, 0) \\
= & \sigma^{-a} \frac{\partial f}{\partial b}(b, 0) \sigma^{a}-\frac{\partial f}{\partial b}(a+b, 0)+\frac{\partial f}{\partial b}(a, b) \\
= & \frac{\partial}{\partial c}\left[\left(\delta^{2} f\right)(a, b, c)\right]_{\left.\right|_{c=0}}
\end{aligned}
$$

So $\frac{\partial g}{\partial b}=0$; hence $g(a, b)$ is constant in $b$. When $b=0$ we have

$$
g(a, 0)=f(a, 0)-\left(\sigma^{-a} h(0) \sigma^{a}-h(a)+h(a)\right)=0 .
$$

So $g \equiv 0$. Because $f$ was chosen arbitrarily we conclude $\mathcal{H}^{2}\left(\mathbb{C} ; C^{\infty}(\operatorname{End}(\tilde{E}))\right)=$ 0 . 
We now proceed with the proof of Proposition 1.2. To show the existence of a family $\left(A_{s}\right)_{s \in \mathbb{C}}$ as stated in the proposition, we will show that there exists a family $\left(A_{(s)}\right)_{s \in \mathbb{C}}$ of pseudodifferential operators that satisfies the conditions of Proposition 1.2 only up to smoothing operators. More precisely:

Proposition 1.4. There exists a holomorphic family of pseudodifferential operators $\left(A_{(s)}\right)_{s \in \mathbb{C}}$ with principal symbols $\sigma_{p r}\left(A_{(s)}\right)=\left(\sigma_{p r}(A)\right)^{s}$ such that $A_{(0)}=I d$, $A_{(1)} \equiv A$ and $A_{(s)} A_{(t)} \equiv A_{(s+t)}$ modulo smoothing operators. This family is unique up to smoothing operators.

Proof. The statement of the Theorem is equivalent to:

$$
\begin{cases}A_{(s)} A_{(t)} A_{(s+t)}^{-1} \equiv I d & \left(\bmod \Psi^{-\infty}\right) \\ A_{(1)} A^{-1} \equiv I d & \left(\bmod \Psi^{-\infty}\right) \\ A_{(0)}=I d & \end{cases}
$$

(we denoted the space of smoothing operators by $\Psi^{-\infty}$ ).

To prove Proposition 1.4, we will construct $A_{(s)}$ inductively in $k \in \mathbb{N}$, such that

$$
\begin{cases}A_{(s)} A_{(t)} A_{(s+t)}^{-1} \equiv I d & \left(\bmod \Psi^{-k}\right), \\ A_{(1)} A^{-1} \equiv I d & \left(\bmod \Psi^{-k}\right), \\ A_{(0)}=I d . & \end{cases}
$$

For $k=1$ we can choose $\left(A_{(s)}\right)_{s \in \mathbb{C}}$ to be a holomorphic family of pseudodifferential operators of order $s$ with the principal symbol equal to $\sigma^{s}$ where $\sigma$ is the principal symbol of $A$. We can construct such a family using a partition of unity. Moreover $A_{(0)}$ can be chosen to be the identity. The operators $A_{(s)} A_{(t)} A_{(s+t)}^{-1}$ and $A_{(1)} A^{-1}$ are operators of order 0 with the principal symbol equal to the principal symbol of the identity. The relations (1.4) are satisfied modulo $\Psi^{-1}$.

Now suppose that the relations (1.4) hold for a certain $k \in \mathbb{N}$. We will construct a new family $\left(\tilde{A}_{(s)}\right)_{s \in \mathbb{C}}$ that satisfies $(1.4)$ for $k+1$ that is of the following form:

$$
\tilde{A}_{(s)}=A_{(s)}\left(I d-H_{(s)}\right), \quad H_{(s)} \in \Psi^{-k} .
$$

In this way $\tilde{A}_{(s)}-A_{(s)} \in \Psi^{s-k}$. We have:

$$
\begin{aligned}
\tilde{A}_{(s)} \tilde{A}_{(t)} \tilde{A}_{(s+t)}^{-1} \equiv & A_{(s)}\left(I d-H_{(s)}\right) A_{(t)}\left(I d-H_{(t)}\right)\left(I d+H_{(s+t)}\right) A_{(s+t)}^{-1} \\
\equiv & A_{(s)} A_{(t)} A_{(s+t)}^{-1}-A_{(s)} H_{(s)} A_{(t)} A_{(s+t)}^{-1}-A_{(s)} A_{(t)} H_{(t)} A_{(s+t)}^{-1} \\
& +A_{(s)} A_{(t)} H_{(s+t)} A_{(s+t)}^{-1} \\
\equiv & I d+F_{(s, t)}-A_{(s)} H_{(s)} A_{(t)} A_{(s+t)}^{-1}-A_{(s)} A_{(t)} H_{(t)} A_{(s+t)}^{-1} \\
& +A_{(s)} A_{(t)} H_{(s+t)} A_{(s+t)}^{-1} \quad\left(\bmod \Psi^{-k-1}\right)
\end{aligned}
$$

where $F_{(s, t)}=A_{(s)} A_{(t)} A_{(s+t)}^{-1}-I d, F_{(s, t)} \in \Psi^{-k}$ by the induction step. To proceed with the induction we have to find a family $\left(H_{(s)}\right)_{s \in \mathbb{C}}$ that makes the right hand side of the equivalence (1.6) equal to the identity modulo $\Psi^{-k-1}$. If $\sigma_{p r}(F(s, t)$ ) and $h(s)=\sigma_{p r}(H(s))$ are the principal symbols, then the condition on $H(s)$ is equivalent to:

$$
\begin{gathered}
\sigma_{p r}(F(s, t))=\sigma^{s} h(s) \sigma^{-s}+\sigma^{s+t} h(t) \sigma^{-(s+t)}-\sigma^{s+t} h(s+t) \sigma^{-(s+t)} \quad \text { or } \\
\sigma^{-(s+t)} \sigma_{p r}(F(s, t)) \sigma^{s+t}=\sigma^{-t} h(s) \sigma^{t}-h(s+t)+h(t)
\end{gathered}
$$


Because both sides are sections in the bundle $\operatorname{End}(\tilde{E})$ over $T^{*}(M) \backslash\{0\}$ of degree of homogeneity $-k$, then the above equality is satisfied if it holds when both sections are restricted to the cosphere bundle $S^{*}(M)$. Let:

$$
f(t, s)=\sigma^{-(s+t)} \sigma_{p r}(F(s, t)) \sigma^{s+t} \quad \text { restricted to } S^{*}(M) .
$$

We will show that $f \in \mathcal{C}^{2}\left(\mathbb{C} ; C^{\infty}(\operatorname{End}(\tilde{E}))\right)$ and $\delta^{2} f=0$. Then $h$ as in (1.7) will be a 1-cochain so that $\delta h=f$.

We would also want the second condition of (1.4) to be satisfied so:

$$
\begin{aligned}
A^{-1} \tilde{A}_{(1)} & \equiv A^{-1} A_{(1)}\left(I d-H_{(1)}\right) \\
& \equiv I d+\left(A^{-1} A_{(1)}-I d\right)-A^{-1} A_{(1)} H_{(1)} \\
& \equiv I d \quad\left(\bmod \Psi^{-k-1}\right)
\end{aligned}
$$

and this holds if

$$
h(1)=\sigma_{p r}\left(A^{-1} A_{(1)}-I d\right)
$$

(we already know that $\left(A^{-1} A_{(1)}-I d\right) \in \Psi^{-k}$ from the induction step).

We will have to show that $f$ is a cocycle in $\mathcal{C}^{2}$. Obviously, $f(0, t)=f(s, 0)=0$. We have:

$$
\begin{aligned}
\left(\delta^{2} f\right)(s, t, r)= & \sigma^{-s} f(t, r) \sigma^{s}-f(s+t, r)+f(s, t+r)-f(s, t) \\
= & \sigma^{-s}\left[\sigma^{-(t+r)} \sigma_{p r}(F(r, t)) \sigma^{t+r}\right] \sigma^{s}-\sigma^{-(s+t+r)} \sigma_{p r}(F(r, s+t)) \sigma^{s+t+r} \\
& +\sigma^{-(s+t+r)} \sigma_{p r}(F(t+r, s)) \sigma^{s+t+r}-\sigma^{-(s+t)} \sigma_{p r}(F(t, s)) \sigma^{s+t}=0
\end{aligned}
$$

is equivalent to

$$
\sigma_{p r}(F(r, t))-\sigma_{p r}(F(r, s+t))+\sigma_{p r}(F(t+r, s))-\sigma^{r} \sigma_{p r}(F(t, s)) \sigma^{-r}=0 .
$$

To see this, consider the following equivalences modulo $\Psi^{-k}$ :

$$
\begin{aligned}
& (I d+F(r, t))(I d+F(t+r, s))(I d-F(r, s+t)) A_{(r)}(I d-F(t, s)) A_{(r)}^{-1} \\
\equiv & A_{(r)} A_{(t)} A_{(t+r)}^{-1} A_{(t+r)} A_{(s)} A_{(s+t+r)}^{-1} A_{(s+t+r)} A_{(s+t)}^{-1} A_{(r)}^{-1} A_{(r)} A_{(s+t)} A_{(s)}^{-1} A_{(t)}^{-1} A_{(r)}^{-1} \\
\equiv & I d
\end{aligned}
$$

and the first term is also equivalent to

$$
I d+F(r, t)-F(r, s+t)+F(t+r, s)-A_{(r)} F(t, s) A_{(r)}^{-1}
$$

which proves (1.10). So $f(s, t)=\sigma^{-(s+t)} \sigma_{p r}(F(t, s)) \sigma^{s+t}$ is a cocycle.

Proposition 1.3 provides us with a family $h(s)$ such that $\delta h=f$. We can choose this family so that (1.9) holds as well. This determines $h$ in a unique way. If $\left(H_{(s)}\right)_{s \in \mathbb{C}}$ is a holomorphic family of pseudodifferential operators of fixed order $-k$ with principal symbol $h(s)$ and $H_{(1)}=I d$, then $\tilde{A}_{(s)}=A_{(s)}\left(I d-H_{(s)}\right)$ satisfies the equivalences (1.4) modulo $\Psi^{-k-1}$.

In this way we obtain a sequence of families of operators $\left(A_{(s)}^{(k)}\right)_{s \in \mathbb{C}}$ that satisfy the relations (1.4) for each $k \in \mathbb{N}$. Moreover, $A_{(s)}^{(k+1)}-A_{(s)}^{(k)} \in \Psi^{s-k}$. Then, using a standard procedure as in Lemma 1.2 .8 in [Gi], we can construct a family $\left(A_{(s)}\right)_{s \in \mathbb{C}}$ whose asymptotic expansion of the total symbol will be equal to:

$$
\sigma_{\text {total }}\left(A_{(s)}\right) \sim \sigma_{\text {total }}\left(A_{(s)}^{(1)}\right)+\sum_{k \geq 0} \sigma_{\text {total }}\left(A_{(s)}^{(k+1)}-A_{(s)}^{(k)}\right)
$$


The family $\left(A_{(s)}\right)_{s \in \mathbb{C}}$ will satisfy the conditions of Proposition 1.4.

$\left(A_{(s)}\right)_{s \in \mathbb{C}}$ is unique up to smoothing operators because it must satisfy the relations (1.4) for all $k \in \mathbb{N}$ and so it must be equal to $\left(A_{(s)}^{(k)}\right)_{s \in \mathbb{C}}$ modulo $\Psi^{-k}$.

Proof of Theorem 1.1 and Proposition 1.2. Once we obtained the family of pseudodifferential operators $\left(A_{(s)}\right)_{s \in \mathbb{C}}$, the proofs of Thm. 1.1 and Prop. 1.2 are identical to the proof of Theorem 5.1 in $[\mathrm{G}]$. We can construct the one parameter group of operators as in Prop. 1.2 using the differential equation:

$$
\dot{A}_{s}=P A_{s} \quad \text { with } \quad A_{0}=I d
$$

where $P=\dot{A}_{(0)}$. If $A_{(s)}$ is made a self-adjoint family in $s$ (i.e., $A_{(s)}^{*}=A_{(\bar{s})}$ ) by replacing it with $\frac{1}{2}\left(A_{(s)}+A_{(\bar{s})}^{*}\right), P$ becomes a self-adjoint operator. By construction $A_{s} \in \Psi^{s}$. Then, using a theorem of Stone (Thms. VIII.7 and VIII.8 [RS]), it can be shown that $A_{s}=\left(A_{1}\right)^{s}$ with $P$ the infinitesimal generator of this one parameter group. In this case:

$$
\left(A_{1}\right)^{s}-A^{s}=\frac{1}{2 \pi i} \int_{\gamma} \lambda^{s}(\lambda-A)^{-1}\left(A-A_{1}\right)\left(\lambda-A_{1}\right)^{-1} d \lambda
$$

and this is a smoothing operator. Because $\left(A_{1}\right)^{s}=A_{s} \in \Psi^{s}$ we obtain $A^{s} \in \Psi^{s}$.

\section{ZETA FUNCTION OF AN ELLIPTIC PSEUDODIFFERENTIAL OPERATOR}

Let $\left(A_{(s)}\right)_{s \in \mathbb{C}}$ be a family of pseudodifferential operators depending holomorphically on the complex parameter $s, A_{(s)} \in \Psi^{s}$. For $\operatorname{Re}(s)<-\operatorname{dim}(M), A_{(s)}$ is a trace-class operator.

Definition 2.1. The trace function of the family $A_{(s)}$ is the holomorphic function $\operatorname{Trace}_{N}\left(A_{(s)}\right)$ defined on the half-plane $\operatorname{Re}(s)<-\operatorname{dim}(M)$.

The von Neumann trace of $A_{(s)}$ is obtained by integrating the von Neumann trace of the Schwartz kernel on $M$ for $\operatorname{Re}(s)<-\operatorname{dim}(M)$. If $A$ is an elliptic positive self-adjoint pseudodifferential operator of order 1 with $\operatorname{Spec}(A) \in(\epsilon, \infty)$, then its zeta function $\zeta_{A}$ is equal to the trace function associated with the family of its complex powers $A^{s}$.

In this section of our paper we will show that $\operatorname{Trace}_{N}\left(A_{(s)}\right)$ has a meromorphic continuation to the whole complex plane with at most simple poles at $-m,-m+1$, $\ldots$, where $m=\operatorname{dim}(M)$. This fact has been proved by Seeley [S]. Guillemin has a different proof in $[\mathrm{G}]$ that applies only for scalar pseudodifferential operators. We will adapt his proof for the case of operators that act on sections in a vector bundle $E$ over the base space $M$.

We start by recalling some definitions and constructions in [G].

Let $\omega$ be the canonical symplectic form on the cotangent space $Y=T^{*}(M) \backslash$ $\{0\}$. The multiplicative group $\left(\mathbb{R}^{+}, \cdot\right)$ acts on $Y$ by multiplication along the fiber $(t,(x, \xi)) \stackrel{\rho}{\rightarrow}(x, t \xi)$. By identifying the groups $\left(\mathbb{R}^{+}, \cdot\right)$ and $(\mathbb{R},+)$ via $\ln : \mathbb{R}^{+} \rightarrow \mathbb{R}$, $\rho$ can be seen as a 1-parameter group of isomorphisms. Let $\Xi$ be the vector field on $Y$ associated with this 1-parameter group and $\alpha=\iota \Xi \omega$ be the contraction of $\omega$ along $\Xi$. Then the $(2 m-1)$-form on $Y, \mu=\alpha \wedge \omega^{m-1}$, is homogeneous of degree $m$, $\rho_{t}^{*} \mu=t^{m} \mu$, and it is horizontal with respect to the fibration $Y=T^{*}(M) \backslash\{0\} \stackrel{\pi}{\rightarrow}$ $S^{*}(M)$.

Let $\mathcal{B}$ be a von Neumann algebra. In our case $\mathcal{B}$ will be the field of complex numbers $\mathbb{C}$, our initial von Neumann algebra $\mathcal{A}$, or the von Neumann algebra of $\mathcal{A}$ 
linear endomorphisms End $\operatorname{A}_{\mathcal{A}}(V)$, where $V$ is a Hilbert module of finite type over $\mathcal{A}$. Let $\overline{\mathcal{P}}_{s}$ be the space of smooth homogeneous $\mathcal{B}$-valued functions defined on $Y$ of degree of homogeneity $s \in \mathbb{C}$ and $\mathcal{P}_{s}$ the space of smooth scalar functions on $Y$ of degree of homogeneity $s$. If $f \in \overline{\mathcal{P}}_{-m}$, then the $\mathcal{B}$-valued $(2 m-1)$ form $f \mu$ is horizontal and invariant under the action of $\left(\mathbb{R}^{+}, \cdot\right)$ so it is of the form $\pi^{*} \mu_{f}$ where $\mu_{f}$ is a $(2 m-1)$-form on $S^{*}(M)$.

Definition 2.2. The residue of $f \in \overline{\mathcal{P}}_{-m}$ is equal to the integral

$$
\overline{\operatorname{Res}} f=\int_{S^{*}(M)} \mu_{f} \in \mathcal{B} .
$$

For $f \in \overline{\mathcal{P}}_{s}, s \neq-m$, we define $\overline{\operatorname{Res}} f=0$. If $\mathcal{B}=\mathbb{C}$, we will denote the residue simply by Res $f$.

Consider the Poisson bracket $\{$,$\} on T^{*}(M)$ associated with the canonical symplectic form $\omega$. Let $\left\{\mathcal{P}_{s}, \overline{\mathcal{P}}_{t}\right\}$ be the space of functions spanned by $\{f, g\}$ with $f \in \mathcal{P}_{s}$ and $g \in \overline{\mathcal{P}}_{t}$. Then $\left\{\mathcal{P}_{s}, \overline{\mathcal{P}}_{t}\right\} \subset \overline{\mathcal{P}}_{s+t-1}$. Following the same method as in [G] (Theorem 6.2), it can be shown that:

a) If $s \neq-m$, then $\left\{\mathcal{P}_{1}, \overline{\mathcal{P}}_{s}\right\}=\overline{\mathcal{P}}_{s}$.

b) If $s=-m$, then $\left\{\mathcal{P}_{1}, \overline{\mathcal{P}}_{s}\right\}$ consists of all functions $f$ for which $\overline{\operatorname{Res}} f=0$.

Moreover, one can construct a family of functions $\left(g_{i}\right)_{i \in I}, g_{i} \in \mathcal{P}_{1}$ such that for any analytic family with parameter $s, f_{s} \in \overline{\mathcal{P}}_{s}$, defined on a strip $a-\epsilon \leq \operatorname{Im}(s) \leq$ $a+\epsilon, c \leq R e(s) \leq d$ for which $\overline{\operatorname{Res}} f_{-m}=0$, one can find $\delta \leq \epsilon$ and homogeneous functions $h_{i, s} \in \overline{\mathcal{P}}$ which are analytic in $s$ on a narrower strip $a-\delta \leq \operatorname{Im}(s) \leq a+\delta$, $c \leq \operatorname{Re}(s) \leq d$, such that

$$
f_{s}=\sum_{i \in I}\left\{g_{i}, h_{i, s}\right\}
$$

(cf. [G], Theorem 6.7).

Let us consider now a holomorphic family of pseudodifferential operators $\left(A_{(s)}\right)_{s \in \mathbb{C}}, A_{(s)} \in \Psi^{s}$ and its associated trace function $\operatorname{Trace}_{N}\left(A_{(s)}\right)$. We define the residue of the family $A$ to be $\operatorname{Res} A=\operatorname{Res}\left(\operatorname{Trace}_{N} \sigma_{p r}\left(A_{(-m)}\right)\right) \in \mathbb{C}$.

We have the following theorem:

Theorem 2.3. The trace function of the analytic family $\left(A_{(s)}\right)_{s \in \mathbb{C}}$ has a meromorphic continuation to the whole complex plane with at most simple poles at $-m,-m+1, \ldots$. The residue of $\operatorname{Trace}_{N}\left(A_{(s)}\right)$ at $s=-m$ is equal to

$$
\operatorname{res}_{\left.\right|_{s=-m}} \operatorname{Trace}_{N}\left(A_{(s)}\right)=\gamma_{0} \operatorname{Res} A
$$

where $\gamma_{0}$ is a constant depending only on $\operatorname{dim}(M)$. For $A_{(s)}=A^{s}$ - the complex powers of an elliptic positive self-adjoint pseudodifferential operator of order one, the residue of the zeta function at $s=-m$ depends only on its principal symbol $\sigma=\sigma_{p r}(A)$ and is equal to $\gamma_{0} \operatorname{Res}\left(\sigma^{-m}\right)$.

Proof. Let $\left(U_{\alpha}\right)_{\alpha}$ be an open cover of $M$ with chosen trivializations of the vector bundle $E$ over each $U_{\alpha}, E_{\left.\right|_{U_{\alpha}}} \cong U_{\alpha} \times V$, with $V$ the generic fiber. Using a partition of unity argument, we can write:

$$
A_{(s)}=\sum_{\alpha} A_{\alpha(s)}+K_{(s)}
$$

where $A_{\alpha(s)}$ are pseudodifferential operators of order $s$ with support inside $U_{\alpha}$ and $K_{(s)}$ is a family of smoothing operators. Because the residue of the trace function 
of the family $A_{(s)}$ and $\operatorname{Res} A$ are both linear in $A$, it is sufficient to prove the theorem for $A_{\alpha(s)}$ and $K_{(s)}$. But $K_{(s)}$ is a family of smoothing operators and both the residues of their trace function and the residue Res $K$ are zero. Thus we reduced the proof of the theorem to the case of one family $A_{(s)}=A_{\alpha(s)}$ supported in an open set $U=U_{\alpha}$ over which we have a trivialization of the vector bundle $\chi_{\alpha}: E_{\mid U} \rightarrow U \times V$. Moreover, because both the trace function $\operatorname{Trace}_{N}\left(A_{(s)}\right)$ and the residue Res $A$ are obtained by integrating quantities that depend on the local expression of the total symbol of $A_{(s)}$, we can replace the bundle $E \rightarrow M$ with the trivial bundle $M \times V \rightarrow M$ and the operators $A_{\alpha(s)}$ with the pseudodifferential operators acting on sections of the trivial bundle $M \times V$ that are supported in the open set $U_{\alpha}$ and equal to $A_{\alpha(s)}$ via the isomorphism $\chi_{\alpha}$. To make things simple, we will denote this new family of operators by $A_{(s)}$ as well, and the new trivial bundle by $E$.

Following the ideas in $[\mathrm{G}]$, we consider the family $(s+m) A_{(s)}$. The principal symbol $(s+m) \sigma_{p r}\left(A_{(s)}\right)$ can be represented by the $\mathcal{B}$-valued smooth homogeneous functions of degree $s, f_{(s)}: T^{*}(M) \backslash\{0\} \rightarrow \mathcal{B}$, with $\mathcal{B}=\operatorname{End}_{\mathcal{A}}(V)$. For $s=-m$ we have $f=0$, so $\overline{\operatorname{Res}} f=0$. Then there exist $\mathcal{B}$-valued functions $h_{(s)}^{k}, h_{(s)}^{k} \in \overline{\mathcal{P}}_{s}$ such that

$$
f_{(s)}=\sum_{k}\left\{g_{k}, h_{(s)}^{k}\right\}
$$

and $h_{(s)}^{k}$ are analytic on a strip $a-\epsilon \leq \operatorname{Im}(s) \leq a+\epsilon, c \leq \operatorname{Re}(s) \leq d$.

Let $G_{k}=G_{k}^{\prime} \hat{\otimes} I d$ be a pseudodifferential operator acting on the space of sections $C^{\infty}(M) \hat{\otimes} V$ of the trivial bundle $E$ with $G_{k}^{\prime}$ a scalar pseudodifferential operator that has the principal symbol equal to $g_{k}$ and $I d$ the identity operator. Let $\left(H_{(s)}^{k}\right)$ be a holomorphic family of pseudodifferential operators with the principal symbol equal to $h_{(s)}^{k}$. Then the principal symbol of the commutator is equal to

$$
\sigma_{p r}\left[G_{k}, H_{(s)}^{k}\right]=\left\{g_{k}, h_{(s)}^{k}\right\}
$$

so

$$
(s+m) A_{(s)}=\sum_{k}\left[G_{k}, H_{(s)}^{k}\right]+B_{(s)} \quad \text { with } B_{(s)} \in \Psi^{s-1} .
$$

For $\operatorname{Re}(s)$ sufficiently small, $\operatorname{Trace}_{N}\left(\left[G_{k}, H_{(s)}^{k}\right]\right)=0$, so $\operatorname{Trace}_{N}\left(A_{(s)}\right)=$ $\frac{1}{s+m} \operatorname{Trace}_{N}\left(B_{(s)}\right)$ for $R e(s)<-m$. But $\frac{1}{s+m} \operatorname{Trace}_{N}\left(B_{(s)}\right)$ is a meromorphic function on the half-plane $\operatorname{Re}(s)<-m+1$ with a simple pole at $s=-m$. So $\operatorname{Trace}_{N}\left(A_{(s)}\right)$ has a meromorphic extension to $\operatorname{Re}(s)<-m+1$. Replacing the family $A_{(s)}$ by $B_{(s)}$ and using an induction argument, we can extend $\operatorname{Trace}_{N}\left(A_{(s)}\right)$ to a meromorphic function on the complex plane with at most simple poles at $-m$, $-m+1, \ldots$.

We will compare the residue of $\operatorname{Trace}_{N}\left(A_{(s)}\right)$ at $-m$ to the residue of the family $\left(A_{(s)}\right)_{s \in \mathbb{C}}, \operatorname{Res} A=\operatorname{Res}\left(\operatorname{Trace}_{N} \sigma_{p r}\left(A_{(-m)}\right)\right)$. Guillemin has showed ([G], Theorem 7.5) that in the scalar case there exists a constant $\gamma_{0}$ that depends only on the dimension of the manifold $M$ such that

$$
\operatorname{res}_{\mid s=-m} \operatorname{Trace} A=\gamma_{0} \operatorname{Res} A \text {. }
$$

We will extend this equality for the pseudodifferential operators acting on sections in the vector bundle $E$. 
We will show a stronger equality:

$$
\text { res }_{\left.\right|_{s=-m}} \overline{\operatorname{Trace}} A=\gamma_{0} \overline{\operatorname{Res}} A_{(-m)}
$$

where $\left(A_{(s)}\right)_{s}$ is a holomorphic family of pseudodifferential operators acting on the sections of the trivial bundle $M \times V$, Trace $A_{(s)}=\int_{M} K_{s}(x, x) d x$ with $K_{s}(x, y)$ the Schwartz kernel of $A_{(s)}$, and $\overline{\operatorname{Res}} A_{(-m)}=\overline{\operatorname{Res}} \sigma_{p r}\left(A_{(-m)}\right)$, both sides of the equality (2.3) being in the von Neumann algebra $\mathcal{B}=\operatorname{End}_{\mathcal{A}}(V)$. The equality $(2.2)$ will be then a direct consequence of (2.3) after passing to the von Neumann traces.

Both sides of the equality (2.3) depend only on the principal symbol of the operator $A_{(-m)}$. This is obvious for the right-hand side. If one considers another family $B_{(s)}$ with $\sigma_{p r}\left(B_{(-m)}\right)=\sigma_{p r}\left(A_{(-m)}\right)$, then $\left(B_{(s)}-A_{(s)}\right)$ is a family for which $\overline{\operatorname{Res}} \sigma_{p r}\left(B_{(-m)}-A_{(-m)}\right)=0$, so, by a previous observation, $\overline{\operatorname{Trace}}\left(B_{(s)}-A_{(s)}\right)$ has a meromorphic extension which is holomorphic at $s=-m$. So $\overline{\operatorname{Trace}} B_{(s)}$ and $\overline{\text { Trace }} A_{(s)}$ will have the same residue at $s=-m$ and this shows that the left-hand side of (2.3) depends only on $\sigma_{p r}\left(A_{(-m)}\right)$.

Both sides of (2.3), as functions of holomorphic families, will factor through the projection $A_{(s)} \rightarrow \sigma_{p r}\left(A_{(-m)}\right) \in \overline{\mathcal{P}}_{-m}$. It will be sufficient to show that the equality (2.3) holds on $\overline{\mathcal{P}}_{-m}$.

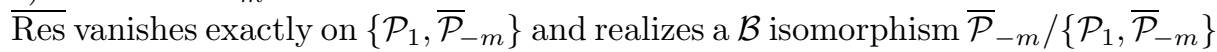
$\stackrel{\sim}{\rightarrow} \mathcal{B}$. For $f \in\left\{\mathcal{P}_{1}, \overline{\mathcal{P}}_{-m}\right\}, f=\sum\left\{g_{k}, h^{k}\right\}$, one can extend it to a holomorphic family of homogeneous symbols of degree of homogeneity $s \in \mathbb{C}$ by considering first the homogenous holomorphic extensions $h_{(s)}^{k} \in \overline{\mathcal{P}}_{s}$ and then taking $f_{(s)}=\sum\left\{g_{k}, h_{(s)}^{k}\right\}$. If $G_{k}=G_{k}^{\prime} \hat{\otimes} I d$ is a pseudodifferential operator such that the scalar operator $G_{k}^{\prime}$ has the principal symbol equal to $g_{k}$ and $\left(H_{(s)}^{k}\right)$ is a holomorphic family of pseudodifferential operators with the principal symbol equal to $h_{(s)}^{k}$, then $A_{(s)}$ defined as $\sum\left[G_{k}, H_{(s)}^{k}\right]$ has the principal symbol at $s=-m$ equal to $f$ and its trace is identically zero. This shows that $\operatorname{res}_{\left.\right|_{s=-m}} \overline{\text { Trace }} A$ vanishes on $\left\{\mathcal{P}_{1}, \overline{\mathcal{P}}_{-m}\right\}$ as well. Because both $\operatorname{res}_{\left.\right|_{s=-m}} \overline{\operatorname{Trace}} A$ and $\overline{\operatorname{Res}} A_{(-m)}$ are $\mathcal{B}$ linear, one gets res $\left.\right|_{s=-m} \overline{\operatorname{Trace}} A=\overline{\operatorname{Res}} A_{(-m)} \cdot C$ with $C \in \mathcal{B}$.

Guillemin already showed this equality for a holomorphic family of scalar pseudodifferential operators $\left(A_{(s)}\right)$ in which case $C$ is a scalar constant $\gamma_{0}$. So $C=\gamma_{0} \cdot \operatorname{Id}_{\mathcal{B}}$ and the equality (2.3) holds. Passing to the von Neumann trace, we get (2.2).

\section{REFERENCES}

[BFKM] D. Burghelea, L. Friedlander, T. Kappeler, P. MacDonald, Analytic and Reidemeister Torsion for Representations in Finite Type Hilbert Modules, Geometric and Functional Analysis 6 (5) (1996), 752-859. MR 97i:58177

[G] V. Guillemin, A New Proof of Weyl's Formula on the Asymptotic Distribution of Eigenvalues, Adv. in Math. 55 (1985), 131-160. MR 86i:58135

[Gi] P. Gilkey, Invariance Theory, the Heat Equation, and the Atiyah-Singer Index Theorem, Publish or Perish, Wilmington, (1984). MR 86j:58144

[RS] M. Reed, B. Simon, Methods of Modern Mathematical Physics, Functional Analysis I, Academic Press, Revised and Enlarged Edition, (1980). MR 85e:46002

[S] R. Seeley, Complex Powers of an Elliptic Operator, Proc. Symp. Pure Math. AMS 10 (1967), 288-307. MR 38:6220

Department of Mathematics, Ohio State University, $231 \mathrm{~W}$ 18th Ave., Columbus, Ohio 43210

E-mail address: bogdanb@math.ohio-state.edu 